Julian Nida-Rümelin

\title{
Verantwortung für internationale Gerechtigkeit: Die globale Herausforderung
}

In: Nida-Rümelin, Julian / Daniels, Detlef von / Wloka, Nicole (Hrsg.): Internationale Gerechtigkeit und institutionelle Verantwortung. - ISBN: 978-3-11-061586-9. - Berlin: De Gruyter, 2019. S. 21-42

(Forschungsberichte / Interdisziplinäre Arbeitsgruppen, Berlin-Brandenburgische Akademie der Wissenschaften ; 41) 


\section{Verantwortung für internationale Gerechtigkeit: Die globale Herausforderung}

\section{Verantwortung: individuell, kollektiv, global}

Wer ist wofür verantwortlich? Wie sind die Kriterien für die individuelle, kollektive oder auch globale Verantwortlichkeit zu fassen? In welchem Verhältnis stehen die unterschiedlichen Verantwortungstypen? Lässt sich jede Form von Verantwortlichkeit auf individuelle Verantwortlichkeit reduzieren? Welche Rolle spielen Institutionen? Sind wir durch die Globalisierung in ökonomischer und kultureller Hinsicht mit einer überbordenden Verantwortlichkeit konfrontiert, der wir als Individuen, möglicherweise aber auch als Einzelstaaten, nicht mehr gerecht werden können? Oder befinden wir uns vielmehr in einer Phase der Weltgeschichte, in der sich politische Verantwortlichkeit auflöst, wir zunehmend in einem System organisierter Unverantwortlichkeit leben (müssen)?

Der Verantwortungsbegriff ist für jede Form der ethischen Stellungnahme unverzichtbar. Wenn Menschen, Kollektive, Staaten für das, was sie tun, keine Verantwortung tragen, dann erübrigt sich jede ethische Beurteilung. Die ethische Beurteilung kommt nicht ex post, sondern ex ante: Es geht um eine Klärung dessen, was wir tun sollen. Wenn es nicht möglich ist, aufgrund der Einsicht in das Richtige zu handeln, wenn Menschen, wie ein zeitgenössischer Feuilleton-Skeptizismus meint, für das, was sie tun, schon deswegen keine Verantwortung tragen, weil es das Ergebnis kausaler Prozesse ist, die sie nicht kontrollieren können, wenn die Eigendynamik von Systemen so ausgeprägt ist, dass Individuen und ihre Praxis keinen Unterschied machen, oder auf globaler Ebene, wenn die Eigendynamik des globalen Wirtschaftssystems jedes einzelstaatliche Agieren zur Makulatur werden lässt, dann verschwinden normative Fragen hinter der bloßen Faktizität. Dann können wir lediglich feststellen, was Sache ist, aber nicht, was zu tun ist.

Verantwortlichkeit ist an die Fähigkeit, Gründe für das eigene Handeln zu geben, gekoppelt. Ohne diese Fähigkeit gibt es keine (Handlungs-)Verantwortung. So agieren wir, so reden wir, so leben wir - dies entspricht unserer Selbstinterpretation. Dieses menschliche Selbstbild, das der anti-normativen Sichtweise entgegensteht, kann nicht einfach ausgetauscht werden, weder aus philosophi-

Detaillierter werden diese Überlegungen in Nida-Rümelin (2017) ausgeführt.

Ә OpenAccess. (C) 2019 Julian Nida-Rümelin, publiziert von De Gruyter. (cc) BY-Nc-SA Dieses Werk ist lizenziert unter der Creative Commons Attribution-NonCommercial-ShareAlike 4.0 Lizenz. 
schen noch aus politischen oder anderen Gründen. Wenn wir das Phänomen der Verantwortung herausbrechen, kollabiert das, was wir unter dem Humanen verstehen, als Ganzes. Die menschliche Lebensform stünde in Frage.

Zwei Einwände drängen sich hier geradezu auf: Ist nicht das, von dem hier die Rede ist, etwas spezifisch Europäisches, ist es nicht an eine bestimmte Kultur gebunden? Und zweitens, ist das Subjekt nicht eine Erfindung, eine Konstruktion der europäischen Aufklärung, die sich über die Verbreitung bestimmter Denkmuster, darunter das der Menschenrechte, unterdessen globalisiert hat? Mit anderen Worten: Ist das, was hier als die menschliche Lebensform (als conditio humana) apostrophiert wird, nichts anderes als ein partikularer Standpunkt, ein kultureller Kontext, eine spezifische Sichtweise, die historisch und kulturell gebunden ist, unter zahlreichen anderen? Diese Einwände sind weit verbreitet, ja sie gehören fast schon zum common sense der Feuilletons und der literarischen Kultur. Sie scheinen mir jedoch ganz offenkundig in die Irre zu gehen.

Ich verstehe unter individueller menschlicher Verantwortung nichts anderes als die Fähigkeit, Gründe abzuwägen, sich von Gründen affizieren zu lassen und Gründe zur Rechtfertigung des eigenen Handelns geltend zu machen. Spricht irgendetwas dafür, dass diese Fähigkeit erst vor rund 300 Jahren, in der Zeit der europäischen Aufklärung, ausgebildet worden ist? Die Antwort muss zweifellos „Nein“ lauten. Nichts deutet darauf hin, dass zu früheren Zeiten diese Fähigkeit, sein eigenes Handeln gegenüber Kritikern zu begründen, nicht existierte. Dass es sich im Laufe der historischen Veränderungen in immer wieder neuen Formen präsentierte, kann man zugestehen, ohne den Kern in Frage zu stellen, nämlich die menschliche Verantwortungsfähigkeit. ${ }^{1}$ Die philosophischen Texte aus der Zeit der griechischen Klassik, diese sind rund 2500 Jahre alt, kann man nicht verstehen, wenn man nicht diese wechselseitige Verantwortungszuschreibung voraussetzt. Die Gesprächspartner in den sokratischen Dialogen tun nichts anderes als Gründe für Überzeugungen, für Handlungen, für Einstellungen (Tugenden) vorzubringen, zu kritisieren, sich also an der Praxis des Gründe-Gebens und Gründe-Nehmens zu beteiligen. Aristoteles entwickelt in seiner Philosophie der Praxis schon einen ausdifferenzierten Verantwortungsbegriff, auch wenn der Terminus als solcher, bezogen auf die Fähigkeit, Antworten zu geben (verANTWORTUNG, responsibility, responsabilità, responsabilité...), wie wir ihn aus den europäischen Sprachen kennen, erst ein spätes Phänomen ist. Auch sehe ich keinerlei Indizien dafür, dass erst mit der Schriftkultur so etwas wie individuelle Verantwortlichkeit aufkommt. Schon deswegen nicht, weil in den meisten historischen Schriftkulturen das

1 Für die These, dass das Phänomen und der Begriff menschlicher Verantwortung erst mit der europäischen Aufklärung auftaucht, vgl. Heidbrink (2003). 
Verfügen über Schrift, der Gebrauch von Schrift, auf eine kleine intellektuelle Elite beschränkt war. Auch sogenannte archaische Kulturen, die es in Rudimenten auch heute noch gibt, kennen die Praxis der Rechtfertigung eigener Handlungen, sie tauschen Gründe aus, sie streiten darüber, ob jemand etwas tun darf oder nicht, sprich: Sie machen sich wechselseitig für das, was sie tun, verantwortlich. Kurz: Weder im historischen noch im interkulturellen Vergleich spricht irgendetwas dafür, dass die Fähigkeit, Gründe für die eigene Praxis abzuwägen und danach zu handeln, sowie das Resultat, nämlich eigene Handlungen gegenüber Kritikern durch das Angeben von Gründen zu rechtfertigen, auf eine spezifische, zum Beispiel europäische, rationalistisch ausgerichtete Kultur beschränkt ist.

Wir sind also verantwortlich, insofern wir die Fähigkeit besitzen, Gründe abzuwägen und aus Gründen heraus zu handeln. Strenggenommen ist dieser Satz trivial, da Handlungen gerade diejenigen Bestandteile unseres Verhaltens sind, die von Gründen geleitet sind. Die vollständig irrationale Handlung gibt es nicht. Wenn wir ein Verhalten als Handlung interpretieren, setzen wir voraus, dass die Person ihr Verhalten unter Kontrolle hatte, dass sie Gründe hatte, sich so zu verhalten und nicht anders. Gründe strukturieren unsere Praxis durch Handlungen. In den Handlungen äußern sich unsere ethischen und empirischen Überzeugungen. Gründe sind dazu da, sicherzustellen, dass die jeweilige Praxis als Ganze in sich stimmig ist und gegenüber Einwänden gerechtfertigt werden kann. Dies gilt für das menschliche Individuum. Gilt dies aber auch für Kollektive, für Staaten, für Institutionen, gilt es auch in der Außenpolitik, in den internationalen Beziehungen, gibt es also so etwas wie globale Verantwortung?

Es hat immer wieder Versuche gegeben, die politische Praxis von der lebensweltlichen $\mathrm{zu}$ entkoppeln. Manche haben dies als das der Politik eingeschriebene Phänomen der schmutzigen Hände beschrieben: Demnach muss erfolgreiche Politik mit ethischen Gründen in Konflikt kommen, sonst handelt es sich nicht um Politik. Viele wenden sich gegen eine Moralisierung des Politischen, da sie eine spezifische Systemlogik in der Politik vermuten, die die Moral außen vor hält. Ähnliches gilt für die ökonomische Praxis. Auch dort ist die Auffassung weit verbreitet, dass Märkte moralfrei seien, dass unternehmerisches Handeln allein auf die Optimierung des Gewinns gerichtet sei und das Handeln von Konsumenten ausschließlich auf die Optimierung des Konsums und dort ethische Aspekte keine Rolle spielen oder jedenfalls nicht spielen sollten. Beides scheint mir offenkundig falsch zu sein (vgl. Nida-Rümelin 2006), das heißt - präziser auf einer gedanklichen Konfusion zu beruhen.

Es gibt keine voneinander isolierten „Systemlogiken“ - eine ökonomische, eine politische, eine lebensweltliche etc. -, sondern vielmehr verschiedene praktische Gründe, auch verschiedene Typen von Gründen, die gegeneinander abgewogen werden müssen, damit sich am Ende daraus eine stimmige, eben 
kohärente Praxis ergibt. Die behauptete Moralfreiheit der Märkte scheitert schon allein daran, dass ohne Kommunikation ökonomisch erfolgreiche Praxis gar nicht möglich ist. Kommunikation aber ist nicht zum moralischen Nulltarif zu haben, denn Kommunikation setzt voraus, dass wir uns in der Regel wahrhaftig und verlässlich äußern, dass wir nur das behaupten, von dem wir selbst überzeugt sind, und versuchen herauszufinden, was tatsächlich der Fall ist (Verlässlichkeit, Realitätsprinzip). Ökonomische Praxis kann nicht erfolgreich sein, wenn Menschen nicht miteinander kommunizieren, zumindest innerhalb eines Unternehmens, aber auch zwischen dem Unternehmen und seinen Kunden. Kooperation ist zum moralischen Nulltarif ebenso wenig zu haben. Die vermeintliche ökonomische Systemlogik der Moralfreiheit ist lediglich die Verabsolutierung eines legitimen Handlungsgrundes, nämlich der Optimierung des Gewinns oder der Optimierung des Konsums. Dieser legitime Handlungsgrund spielt in der ökonomischen Praxis eine große Rolle, aber er muss abgewogen werden gegen andere Handlungsgründe. Geschieht dies nicht, dann erodiert die ökonomische Praxis selbst. Mit anderen Worten: Sie lebt von Bedingungen, die sie auf sich selbst gestellt nicht garantieren kann, um ein Diktum des Verfassungsrichters Böckenförde abzuwandeln.

Ähnliches gilt für die politische Praxis. Auch dort gibt es spezifische Typen von Gründen, die zum Beispiel darauf beruhen, dass die kollektive Meinungsbildung eine große Rolle spielt und es daher ohne die Bereitschaft, Kompromisse zu finden, in der Regel keinen politischen Erfolg gibt. Entsprechend müssen Abstriche gegenüber eigenen Zielen gemacht werden, um kompromiss- und kooperationsfähig zu sein. Moralisierung im Sinne einer rigiden Verabsolutierung einzelner Bewertungsaspekte ist in der politischen Praxis in der Tat hinderlich. Dies heißt aber nicht, dass ethische Gründe keine Rolle spielen. Im Gegenteil, eine politische Praxis, die nicht in der Lage ist, sich nachvollziehbar und ethisch adäquat zu rechtfertigen, scheitert zumindest in einer Demokratie an einer kritischen Öffentlichkeit.

Das generelle Muster von Kooperation, auch außerhalb von Institutionen, lässt Individuen gemeinsam handeln, indem sie ihren Teil zu einer gemeinsam erwünschten Praxis beitragen und dabei auf die Optimierung ihrer eigenen Interessen verzichten. Anders ist Kooperation nicht zu haben (Nida-Rümelin 2011, S. 77-98). In der institutionell verfestigten Form, zum Beispiel im Rahmen der Bundesregierung, muss jede einzelne Ministerin und jeder einzelne Minister so agieren, dass der eigene Beitrag sich zu einem kohärenten Ganzen fügt, der die Handlungsfähigkeit der Institution Bundesregierung sicherstellt. Ist dies nicht der Fall, kommt es zu einer institutionellen Krise, und sei es nur in der vorübergehenden Form des Rücktritts eines Ministers. Der Übergang zwischen politischer Praxis im Rahmen fest gefügter Institutionen, wie zum Beispiel einer Bundesre- 
gierung, und der politischen Praxis als einer institutionell nicht gefestigten Form der Kooperation ist fließend. Kollektive Verantwortung ist eine spezielle Form kooperativer Verantwortung. Politische Verantwortung ist eine spezielle Form kollektiver Verantwortung. Sie ist in unterschiedlichem Maße institutionell verfestigt und beruht auf einer institutionellen Struktur, die kollektives Handeln reguliert. Unabhängig vom Maß der Institutionalisierung tragen die einzelnen Akteure eine individuelle kooperative Verantwortung.

Es gibt also keinen Ausweg aus der Verantwortlichkeit. Zur conditio humana, zur menschlichen Lebensform, die wir über alle Kulturen und Zeiten teilen, gehört die Fähigkeit, Gründe abzuwägen und entsprechend $\mathrm{zu}$ handeln. Wir sind für unsere Praxis verantwortlich. Dies gilt individuell, aber auch kollektiv und politisch. Wie unübersichtlich die Handlungsbedingungen auch immer sind, die einzelnen Akteure formen sich über eine kooperative Praxis zu Verantwortungsgemeinschaften, ob sie es wollen oder nicht. Die globale Dimension macht die Reichweiten, die Wirksamkeiten einzelner Aktionen größer und unübersichtlicher, hebt aber menschliche Handlungsverantwortung nicht auf. Die politische, zumal die demokratische Praxis ist darauf gerichtet, die Gestaltungskraft durch Regelsetzung und Institutionen zu wahren. Die demokratische Ordnung beruht auf der Idee, dass zwar nicht je individuell, aber kollektiv, durch Verständigung auf bestimmte Entscheidungsverfahren, eine Kontrolle zumindest der Regeln möglich ist, die unser Zusammenleben gestalten. Dies gilt in der Kommune, in der Region, auf nationalstaatlicher Ebene, in der EU und auf der ganzen Welt. Insofern gibt es hier eine Korrespondenz zwischen globaler politischer Verantwortlichkeit und der kosmopolitischen Perspektive, also der Institutionalisierung von Politik im globalen Maßstab.

\section{Kommunitarismus versus Kosmopolitismus}

In der politischen Philosophie der Gegenwart stehen sich kommunitaristische und liberalistische Ansätze gegenüber. Tatsächlich hatte die Renaissance der Ethik und der politischen Philosophie mit der Publikation einer Theorie der Gerechtigkeit von John Rawls 1971 begonnen, und diese verstand sich als liberalistisch. $^{2}$ Sie versuchte, die normativen Grundlagen einer liberalen, westlichen, ökonomisch entwickelten Demokratie unter Rückgriff auf das sogenannte Ver-

2 John Rawls präsentiert die politische Essenz seiner Theorie, nun stärker politiktheoretisch als philosophisch, in seinem späten Werk Political Liberalism, in dem auch eine - implizite - Antwort auf die kommunitaristische Herausforderung gegeben wird (Rawls 1993). Die Entwicklung des Rawls'schen Denkens lässt sich in seinen Collected Papers (Rawls 2001) nachlesen. 
tragsargument herauszuarbeiten: gleiche maximale individuelle Freiheiten und eine gesellschaftlich-wirtschaftliche Kooperation, deren Früchte so verteilt werden, dass sie in besonderem Maße den Schlechtergestellten zugutekommen (das sogenannte Differenzprinzip). John Rawls hatte Gerechtigkeit als das Ergebnis der fairen Wahl von Grundprinzipien bestimmt. Fair ist die Wahl dann, wenn man von allen Besonderheiten individueller Interessen abstrahiert. Zugleich aber hat Rawls an der Idee festgehalten, dass rationale Individuen ihre Interessen wechselseitig desinteressiert - verfolgen, und das heißt, nach den Bedingungen eines selbstbestimmten Lebens streben, wozu Einkommen, Chancen und Freiheiten sowie die sozialen Bedingungen der Selbstachtung gehören.

Kommunitaristen wie Michael Sandel haben dagegen eingewandt, dass Individuen als isolierte und wechselseitig desinteressierte gar nicht vorstellbar seien, dass erst die Gemeinschaftszugehörigkeit die (moralische) Person ausmache (Sandel 1982). Die ganze Konstruktion liberalistischer Ethik könne nicht überzeugen. Es gebe keine unencumbered selves. Wir können auf die Details dieser Auseinandersetzung hier nicht eingehen. Wesentlich für das Folgende ist lediglich, dass man den Gegensatz zwischen kommunitaristischer und liberalistischer politischer Philosophie erfasst. Kommunitaristen argumentieren aus der Gemeinschaftszugehörigkeit heraus. Die elaborierteste Form liegt meines Erachtens in Gestalt des Werkes von Michael Walzer vor (Walzer 1983). Hier wird in großer Differenziertheit auf die ganz unterschiedlichen Normen und Werte eingegangen, die die verschiedenen Gemeinschaften prägen. Der ökonomische Markt gehorcht anderen Regeln als die öffentliche Verwaltung. Die Zugehörigkeit zu Vereinen wiederum folgt anderen Regeln als die Zugehörigkeit zu einer Familie. Diese unterschiedlichen Gemeinschaftsformen können, das ist das Credo der Kommunitaristen, nicht von außen, von einem vermeintlich objektiven ethischen Standpunkt aus beurteilt werden, sondern nur aus der Perspektive einer spezifischen Gemeinschaftspraxis. Man bleibt gewissermaßen immer Teil von Gemeinschaften und löst sich nie, wie die Liberalisten annehmen, aus allen gesellschaftlichen Zusammenhängen heraus. Auch die politische Philosophie oder Ethik kann das nicht leisten. Die kommunitaristische Kritik richtet sich gegen die Reduktion moralischer Komplexität, gegen die Vorstellung, es gebe lediglich einige wenige für alle verbindlichen Individualrechte, aus denen sich alles andere herleiten ließe, wie die radikalsten Vertreter des Liberalismus, die Libertären, meinen. Die Kritik der Kommunitaristen verweist zu Recht darauf, dass das Zusammenleben der Menschen zu komplex sei, um es in dieser Weise in ein einziges Schema pressen zu können. Auch der ökonomische Markt hat keine Sonderstellung, er ist eine von vielen unterschiedlichen Formen zwischenmenschlicher Interaktion. Die kommunitaristische Kritik richtet sich aber nicht nur gegen die unzulässige Vereinfachung normativer Aspekte, sondern auch gegen den Uni- 
versalismus, für den die universellen Normen und Werte existieren, die zudem für alle gleichermaßen verbindlich sind.

Der Kommunitarismus geht mit dem (kulturellen) Relativismus eine Verbindung ein. Das Projekt der philosophischen oder ethischen Klärung der Normen und Werte des menschlichen Zusammenlebens wird in die Beschreibung unterschiedlicher Lebensformen überführt. Das Normative verschwindet hinter dem Empirischen. Diejenigen, die auch normative Fragen für rational klärbar halten, befürworten in der Regel eine universalistische Ethik und politische Philosophie. Diejenigen, die normative Fragen grundsätzlich für nicht rational klärbar halten, lehnen universalistische Ethik und politische Philosophie in der Regel ab. Da Migration ein globales Phänomen ist und sich die dadurch aufgeworfenen Fragen nicht auf lokale Gemeinschaftszugehörigkeiten beschränken lassen, spricht viel für eine universalistische Behandlung dieser Thematik. In der Tat werden wir eine kosmopolitische Perspektive einnehmen, das heißt, die Ethik der Migration unter dem Aspekt einer humanen Gestaltung der Weltverhältnisse diskutieren und nicht aus einer nationalstaatlichen oder gruppengebundenen Perspektive. Allerdings wird sich zeigen, dass der hier vertretene Kosmopolitismus den berechtigten Einwänden kommunitaristischer Kritiker durchaus gerecht werden kann. Ein vernünftiger Kosmopolitismus muss mit Gemeinschaftsbindungen unterschiedlichster Art verträglich sein.

Dieser Konflikt zwischen der lokalen Gemeinschaft und ihren ethischen Bindungen einerseits und einer kosmopolitischen, weltbürgerlichen Einstellung und Lebensform andererseits zieht sich durch die Kulturgeschichte seit der Antike. Der Kosmopolit (cosmopolites) der griechischen Klassik erschien seinen Mitbürgern in der Stadt als verantwortungslos, denn er beteiligte sich nicht an den gemeinschaftsbildenden Praktiken der Athener Bürgerschaft, er nahm die Belange der Stadt (ta politika) nicht ernst, wirkte auf seine Zeitgenossen arrogant und distanziert. Dieses Grundmuster hat sich bis heute nicht verändert. Die kosmopolitische Lebensform ist weitgehend ${ }^{3}$ auf die oberen Mittelschichten und Oberschichten der Industriegesellschaften beschränkt, mit Brückenköpfen in den Eliten der Länder des globalen Südens. Man jettet von Ort zu Ort, man verbringt einen Teil seines Lebens in Hotels, die Oberschicht unterhält an verschiedenen Orten eigene Wohnungen und Häuser, die in der Abwesenheit beschützt und gepflegt werden müssen, man hat eine Yacht, die an unterschiedlichen Orten vor

3 So gehört es unterdessen zur Normalbiographie der Studierenden, Auslandsemester einzubauen oder zwischen Schule und Studium Monate oder auch Jahre im Ausland zu verbringen. Dieses Phänomen ist nicht mehr auf die Oberschichten beschränkt. Erst recht gilt das für die Aussteiger und Arbeitsmigranten. Ortsfest sind hingegen ganz überwiegend die kleinbürgerlichen und traditionellen Arbeitermilieus. 
Anker geht und gelegentlich transferiert werden muss, um den Urlaubswünschen ihres Besitzers gerecht zu werden. Man spricht das globalisierte Englisch, das zur internationalen Verkehrssprache in weiten Teilen der Welt geworden ist, erledigt einen Teil seiner Korrespondenz ebenfalls in dieser Sprache und pflegt ökonomische und kulturelle Kontakte in unterschiedliche Weltgegenden. Diese Lebensform geht oft damit einher, dass die betreffenden Personen keine über die Kernfamilie hinausgehenden Bindungen eingehen. Sie engagieren sich nicht in der lokalen Gemeinschaft, weder in der Kirchengemeinde noch im Stadtparlament, sie kümmern sich nicht um die Angelegenheiten des Gemeinwesens, das sie nur für ihre partikularen Interessen in Anspruch nehmen. Wenn Konflikte auftreten, delegieren sie diese an Anwälte. Die direkte Kommunikation ist zu oberflächlich, zu sporadisch, um personale Bindungen jenseits des engsten Freundeskreises aufzubauen.

Die kosmopolitische Lebensform wirkt aus der Perspektive der Gebundenen, der örtlich Verwurzelten, der vielleicht sozioökonomisch Schlechtergestellten, egozentrisch und bindungslos. Die globale „kosmopolitische“ Praxis beruht auf einer Handvoll geteilter Normen und Werte, denen aber die kulturelle Tiefe und die historische Verankerung lokaler und kultureller Gemeinschaften abgeht. Der ökonomische Markt ist der bevorzugte Rahmen globalisierter Interaktionen. Dabei zählt die Möglichkeit, seinen Hauptwohnsitz, unter Umständen auch seine Staatsangehörigkeit, im Laufe des Lebens vielleicht sogar mehrfach zu wechseln, zu den avancierten Formen kosmopolitischer „gehobener“ Existenz. Manche Staaten bieten entsprechende Pakete an: Arbeitsplätze schaffen, hinreichend investieren, den neuen Hauptwohnsitz anmelden, dann lässt sich die Staatsbürgerschaft häufig legal erwerben. Ulrich Beck hat die Kosmopolitisierung der Weltgesellschaft freundlicher beschrieben, nämlich als Prozess der Überlagerung und Vermischung kultureller Identitäten (Beck 2004; Beck/Beck-Gernsheim 2011). Dieses Spannungsverhältnis bleibt aber auch in dieser freundlicheren Perspektive bestehen. Der soziologische Kosmopolitismus löst Strukturen auf, die für viele Menschen, insbesondere für die ökonomisch Schlechtergestellten, Sicherheit und Halt stiften, vor allem aber Gemeinschaftsbildung erst ermöglichen, dazu gehört auch die Sozialstaatlichkeit. Michael Walzers Kritik an den vier Mobilitäten der modernen liberalen Gesellschaften - der familialen (Heirat, Scheidung, Wiederverheiratung, Patchworkfamilien etc.), der lokalen (Wechsel des Wohnsitzes, wie er in den USA um ein Vielfaches häufiger vorkommt als in Europa), der sozialen (Verlassen des eigenen sozioökonomischen Herkunftsmilieus und Wechsel nach oben und zunehmend auch nach unten) und der weltanschaulichen Mobilität (Wechsel der Religionsgemeinschaften, der Zugehörigkeit zu Weltanschauungsgruppen etc.) - beruht darauf, dass diese zwar zu den individuellen Rechten und Freiheiten, die die liberalen westlichen Gesellschaften bieten, gehören, sie aber 
zugleich die Gemeinschaftsbindungen und den gesellschaftlichen Zusammenhalt bedrohen, sodass diese Freiheiten, wenn sie im Übermaß genutzt werden, zur Erosion aller Bindungskräfte führen.

Ein wesentliches Movens der multidimensional mobilen Gesellschaft ist der ökonomische Impetus, also das Bestreben, ökonomische Vorteile zu nutzen, umzuziehen, wenn das Preis-Leistungs-Verhältnis einer anderen Wohnung etwas günstiger ist, den Job zu wechseln, wenn das Einkommen ein wenig höher ist, die Religionsgemeinschaft auszutauschen, wenn ein entsprechend charismatischer Propagandist in Erscheinung tritt, die Partnerin zu wechseln, wenn eine Jüngere und Schönere Interesse zeigt usw.

Paradoxerweise argumentieren diejenigen, die offene Grenzen befürworten, meist kommunitaristisch: Sie meinen, dass auch offene Grenzen nur in geringem Maße zur Verstärkung der Migration beitragen würden, und auf die Nachfrage, wie dies denn angesichts der dramatischen Wohlstandsunterschiede der Welt zu erklären sei, sprechen sie von den lokalen und kulturellen Bindungen, die Menschen nur ungern aufgeben würden. Wir werden darauf noch ausführlicher eingehen, aber hier sei schon einmal festgehalten, dass das liberalistische Modell des ungebundenen Selbst, das seine eigenen Interessen optimiert, nur den globalen Markt als Weltordnung befürworten kann: Alle Staatlichkeit, alle Gemeinschaftsbindung, alle kulturellen Identitäten stünden damit zur Disposition. Man kann die liberalistische - oder besser: libertäre - Vision einer mobilen, durch das universelle Prinzip ökonomischer Optimierung gestalteten Welt nicht kommunitaristisch begründen. Wer diese Welt will, muss auch ihren politischen, kulturellen und sozialen Preis berücksichtigen. Michael Walzer, ein mitunter von der israelischen Kibbuzbewegung geprägter linker Intellektueller, später Herausgeber des führenden kritischen US-Journals Dissent, hatte früh für die Zulässigkeit von Kriegen aus einer kommunitaristischen Begründung argumentiert (Walzer 2015). Im Kapitel „Membership“ seiner Sphären der Gerechtigkeit meint er, dass jede Gemeinschaft grundsätzlich das Recht habe, zu bestimmen, wer zu ihr gehört. Das Recht auf Freizügigkeit wird demnach nicht nur zwischen Staaten, sondern auch zwischen Kommunitäten durch das Recht auf kollektive Selbstbestimmung der Gemeinschaften eingeschränkt.

Der konservativ-katholische Kommunitarist Alasdair MacIntyre schließlich hält es für ausgeschlossen, dass sich aus einer liberalen und universalistischen Sicht Patriotismus überhaupt rechtfertigen lässt. Patriotismus besteht in einer besonderen Loyalität zum eigenen Staat, ohne den ein Staat gar nicht existieren könne, meint MacIntyre, aber diese besondere Loyalität lasse sich nicht begründen, wenn die Zugehörigkeit etwas letztlich moralisch Irrelevantes wäre, wenn dem aus der Perspektive des jeweiligen Staatsbürgers nicht etwas Beson- 
deres zukäme, was diese Gemeinschaft von allen anderen Gemeinschaften unterscheidet (MacIntyre 1995, S. 84-102).

Für den liberalen, eine universalistische Ethik vertretenden Kosmopoliten müssten demnach die ethischen Fundamente der politischen Ordnung zerfallen. Auch der bedeutendste zeitgenössische Vertreter eines normativ begründeten politischen Nationalismus, David Miller, argumentiert kommunitaristisch, mit den besonderen Bindungen einer Nation, die im günstigsten Fall eine staatliche Form hat (Miller 1995). Die kulturelle Zusammengehörigkeit, die gemeinsame Sprache, die gemeinsame Geschichte, die gemeinsame Handlungsfähigkeit konstituieren den Nationalstaat. Und dieser ist nur aufrechtzuerhalten, wenn er auch seine Grenzen kontrolliert.

Tatsächlich sehe ich aber keinen Konflikt zwischen universalistischer Ethik und kosmopolitischer Philosophie mit unseren, nennen wir sie im Folgenden zusammenfassend partikularen Pflichten. Diese Kollision kommt nur zustande, wenn die universalistische Ethik allzu primitiv ist und etwa ein einziges - zum Beispiel utilitaristisches - Prinzip zugrunde liegt, das der Komplexität praktischer Gründe nicht gerecht werden kann. Dies lässt sich am folgenden Beispiel gut erläutern: Wir haben alle ein gemeinsames Interesse daran, dass Eltern für ihre Kinder sorgen, Lehrerinnen für ihre Schüler Verantwortung übernehmen, Lebenspartner und Freunde füreinander einstehen etc. Wir wünschen uns, in einer Gesellschaft zu leben, die dieses Merkmal aufweist, oder anders formuliert: Wir wünschen uns eine je individuelle Praxis, die die Strukturen der Verantwortungswahrnehmung und Pflichterfüllung dieser genannten Arten bewahrt. Die je punktuelle Optimierung würde diese Strukturen sprengen und Humanitätsverluste mit sich führen.

Dieser schlichte Gedanke der Einbettung, oder etwas anspruchsvoller formuliert, der strukturellen Rationalität, nach der wir uns ohnehin in unserer Praxis ausrichten, die aber in der praktischen Philosophie der Gegenwart nur unzureichend berücksichtigt ist, hebt die vermeintliche Unvereinbarkeit kommunitärer und anderer partikularer Bindungen mit universalistischer Ethik und kosmopolitischer Philosophie auf (Nida-Rümelin 2018). Wir müssen uns dann die Mühe machen, zu bestimmen, welche Praktiken als strukturell rational gelten können. Diese Beurteilung erfolgt nicht von außen nach innen: Wir können nicht lediglich eine ideale Welt kosmopolitischer Harmonie entwerfen und von dieser ausgehend bestimmen, wie die einzelne Person zu leben hat.

Wenn das, was aus der globalen Perspektive sinnvoll erscheint, für die individuellen Lebensformen unzumutbar wird, dann muss auch wieder rückwirkend das kosmopolitische Ideal überprüft werden. Der Utopismus der frühen Neuzeit hatte diese gefährliche Schlagseite: zunächst losgelöst von dem, was wir in unserer Lebenspraxis für akzeptabel halten, zu bestimmen, was eine ideale, har- 
monische, wohlgeordnete Gesellschaft beziehungsweise einen solchen Staat ausmacht, um dann die Mittel zu bestimmen, die über Erziehung, Gängelung, Sanktionierung, Umerziehung, etc. den Einzelnen in die Lage versetzen, dieses Ideal in seiner konkreten Lebensform zu realisieren. Die Inhumanität des Utopismus hat ihren Ursprung in einer rationalistischen philosophischen Methode. Das Scheitern der kommunistischen Weltbewegung ist auch Ausdruck einer verfehlten rationalistisch motivierten Politik.

Die zeitgenössische Philosophie muss diese rationalistische Schlagseite vermeiden und der Versuchung des Utopismus entgehen. Sie steht nicht außerhalb aller kultureller Praxis, sondern muss anschlussfähig bleiben, sie systematisiert Gründe, die uns vertraut sind, sie konstruiert keine prinzipiengeleiteten Systeme menschlichen Handelns, sie akzeptiert die Bindungen und Projekte, die eine humane (Welt-)Gesellschaft ausmachen, und integriert diese in eine kosmopolitische Perspektive.

\section{Internationale Gerechtigkeit: Die globale Herausforderung}

Ein berühmter Artikel von Thomas Nagel aus dem Jahr 2005 beginnt mit der These, dass die Welt, wie sie heute ist, nicht gerecht sei, und dass das schwerlich bestritten werden könne (Nagel 2005, S.113-147). Ich stimme Thomas Nagel völlig zu und benenne zunächst einige Aspekte, die für diese These sprechen:

Die Welt ist nicht gerecht, weil viele Millionen Menschen chronisch unterernährt sind, obwohl die Agrarwirtschaft weltweit deutlich mehr produziert als für die Deckung der Ernährungsbedürfnisse der gesamten Weltbevölkerung notwendig wäre. ${ }^{4}$ Es hat sich schlicht als falsch herausgestellt, dass das Bevölkerungswachstum, wie zum Beispiel vom Club of Rome Anfang der 1970er Jahre prophezeit, zu einer immer stärker zunehmenden Nahrungsmittelknappheit führen müsse (angesichts der Begrenztheit der Anbauflächen und ihre Zurückdrängung durch die Ausbreitung menschlicher Siedlungen). Es ist auch nicht primär die Industrialisierung der Landwirtschaft, die es ermöglicht, auf begrenzter Fläche die Ernährungsbedürfnisse der Weltbevölkerung zu befriedigen,

42015 hatten 795 Millionen Menschen nicht genug zu essen, 98\% von ihnen lebten in Entwicklungsländern (Food and Agriculture Organization 2015). Allerdings wächst die weltweite Agrarproduktion schneller als die Bevölkerung. 2014 wurden 2,566 Milliarden Tonnen Getreide geerntet (Food and Agriculture Organization 2016), wovon laut Weltagrarbericht 2013 nur $43 \%$ als Lebensmittel diente (Weltagrarbericht 2013, S. 4). 
vielmehr tritt Hunger meist als Begleitphänomen von Kriegen und Bürgerkriegen auf, als Folge der zynischen Politik lokaler Eliten, die sich über die Bedürfnisse der Bevölkerung und speziell der Landbevölkerung hinwegsetzen, aber auch als Folge drastischer Preissteigerungen von Grundnahrungsmitteln auf den regionalen und globalen Märkten. ${ }^{5}$ Auch wenn der Zusammenhang umstritten ist, ${ }^{6}$ scheint es mir doch auf der Hand zu liegen, dass die zunehmende Praxis der Spekulation im globalen Lebensmittelhandel zur Instabilität der Preisentwicklung beigetragen hat. Starke Preissteigerungen schließen einen Teil der ärmsten Bevölkerung in den Elendsregionen der Welt vom Zugang zu Grundnahrungsmitteln aus und führen zu chronischer Unterernährung und zum täglichen Tod. ${ }^{7}$ Aber auch der drastische Preisverfall von Nahrungsmitteln, die im globalen Süden produziert werden, ${ }^{8}$ kann der kleinteiligen Landwirtschaft dort die ökonomische Basis entziehen, Landflucht ist die Folge, das Fehlen von Arbeitskräften in der oft noch stark von Subsistenzwirtschaft geprägten, kleinteiligen, bäuerlichen Praxis lässt Flächen brachliegen und entzieht der Nahrungsmittelproduktion vor Ort wichtige personelle und natürliche Ressourcen.

5 So sind z. B. die Preise für Mais von $2 \$$ pro Büschel (2002) auf 8 \$ pro Büschel (2012) gestiegen. Weizen erreichte 2012 mit 9 \$ einen Höchststand und konnte einen Zuwachs von 3 \$ in den letzten sieben Jahren verzeichnen. Seit 2013 fallen die Nahrungsmittelpreise insgesamt (Food and Agriculture Organization 2015, 2016). Interessant ist hier auch, dass in den LDCs (least developed countries) die durchschnittlichen Ausgaben für Nahrungsmittel bei $60-80 \%$ des verfügbaren Einkommens liegen, während sie in den Industrieländern 10-20\% ausmachen (World Food Programme 2013).

6 Vgl. Die Kontroverse zwischen foodwatch (Thilo Bode) und der Finanzwirtschaft (GoldmannSachs).

72015 waren 11\% der Weltbevölkerung (795 Millionen Menschen) unterernährt, während 1990 - 92 noch 19\% unterernährt waren (1,011 Millionen Menschen) (World Bank 2017). Unterernährt bedeutet, dass die Person ihren Minimum-Energiebedarf kontinuierlich nicht decken kann; hier liegt er 2,5\% unter dem Mindestmaß. Die Kindersterblichkeit bei Kindern unter 5 Jahren fiel in den vergangenen 25 Jahren um 53\% von 91 Sterbefällen pro 1000 Lebendgeburten (1990) auf 43 pro 1000 (2015) (UNICEF/WB/WHO/UN 2015, S. 1). Trotzdem sind 156 Millionen Kinder unter 5 Jahren Hunger und Umweltproblemen ausgesetzt (child stunting: Resultat chronischer Unterernährung und schlechter Umweltbedingungen); wovon 60 Millionen in Afrika und 59 Millionen in Südostasien leben (World Health Organization 2016, S. 84). 2015 sind 36\% der Sterbefälle von Kindern unter 5 Jahren auf Unterernährung zurückzuführen und $80 \%$ der Neugeborenen-Sterbefälle erklären sich aufgrund eines zu geringen Geburtsgewichts (UNICEF/WB/ WHO/UN 2015, S. 8).

8 Meist können Kleinbauern gar nicht am globalen Markt, und nicht einmal am regionalen Markt anbieten, da ihnen der Zugang (Straßen etc.) fehlt. Es mangelt auch an Düngemittel, um ihre Produktion und ihre Erträge steigern zu können. Wenn die Kleinbauern hier Unterstützung erhielten, könnten sie praktisch von steigenden Lebensmittelpreisen profitieren (Bundesministerium für wirtschaftliche Zusammenarbeit und Entwicklung 2015). 
Die Industrialisierung der Landwirtschaft und der Einsatz grüner Gentechnik, oft als Lösung für die Ernährungssituation angepriesen, sind zumindest ambivalent. Zwar kann die intensivere Nutzung der Böden die Nahrungsmittelproduktion ertragreicher werden lassen, zugleich aber führt sie dazu, dass viele Menschen ihre wirtschaftliche Existenz verlieren und die Subsistenzwirtschaft, die in weiten Teilen des globalen Südens nach wie vor für viele Menschen unverzichtbar ist, erodiert und teilweise massive Schäden auftreten. Dass es trotz des starken weltweiten Wirtschaftswachstums noch immer nicht gelungen ist, den Hunger in der Welt auszurotten, ist ein Armutszeugnis, und ich füge hinzu: Es zeigt die Ungerechtigkeit der Welt deutlich auf.

Die Welt ist ungerecht, weil ein Großteil der Weltbevölkerung im Elend verharrt, obwohl dieses Elend durch fairere Kooperationen weltweit zu beheben wäre. Je nach Kriterium für Elend sind davon rund zwei Milliarden Menschen betroffen. Hier geht es nicht um die zweifelhaften Kriterien von Armutsgefährdung oder relativer Armut, wie sie den Armuts- und Reichtumsberichten, etwa der Bundesregierung, zugrunde liegen, wonach die Armut am Prozentsatz des Medianeinkommens gemessen wird, mit dem paradoxen Effekt, dass heute Menschen, die das doppelte durchschnittliche Realeinkommen der 1960er Jahre zur Verfügung haben, als arm oder armutsbedroht gelten. Allein die Tatsache, dass eine Gruppe oberhalb des Medianeinkommens zusätzliches Einkommen erwirbt, während sich an der Einkommensverteilung nichts verändert, führt nach diesem (relativen) Kriterium zu einem Anstieg der Armut. Nein, es geht im Folgenden nicht um solche Armutskonstrukte, sondern um reale Armut, die sich darin ausdrückt, dass Menschen zum Beispiel von einer Kaufkraft von zwei US-Dollar am Tag leben müssen. Dies ist ein ökonomisches Kriterium von absoluter Armut, das den Usancen internationaler Vergleiche entspricht.

Es ist aber auch möglich, und es wird zunehmend praktiziert, dass man Armut nicht nur in verfügbarer Kaufkraft misst, sondern nach der Verfügbarkeit von Grundgütern, die für ein menschenwürdiges Leben unverzichtbar sind. Dazu gehört der Zugang zu elementaren Bildungseinrichtungen, die Möglichkeit, sich hinreichend gut zu ernähren, zu kleiden und ein Dach über dem Kopf zu haben, für andere sorgen zu können, etwa für eigene Kinder oder die alt gewordenen Eltern und Großeltern, und schließlich eine medizinische Grundversorgung. Diese weicheren Kriterien sind schwieriger zu messen, werden dem Armutsphänomen aber gerechter. ${ }^{9}$ Erneut scheint auch hier folgender Zusammenhang mit der Ge-

9 Der MPI (Multidimensional Poverty Index) wurde 2011 an der Universität Oxford von Sabina Alkire und James Foster für das Entwicklungsprogramm der Vereinten Nationen entwickelt und umfasst die Kategorien Bildung, Gesundheit und Lebensstandard. Der HDI (Human Development Index) ist ein Wohlstandsindikator für Staaten, dessen Entwicklung Amartya Sen 1990 anregte, 
rechtigkeitsfrage zu bestehen: Wenn diese Armut, dieses globale Elend, das rund ein Drittel der Weltbevölkerung betrifft, unvermeidbar wäre, würden wir es beklagen, aber nicht als Ungerechtigkeit bezeichnen. Nichts spricht aber dafür, dass es unvermeidbar ist. ${ }^{10}$ Wenn wir behaupten, das Elend sei vermeidbar, dann legen wir uns auf die These fest, der zufolge es Möglichkeiten gibt, es zu mindern oder zu beseitigen. Für Teilaspekte hierzu gibt es Schätzungen und Berechnungen. Wie viel würde es kosten, bestimmte Seuchen, wie etwa Malaria, weltweit auszurotten ${ }^{11}$ Wie viel würde es kosten, alle Menschen mit täglichem Trinkwasser zu

weil er das vorherige Kriterium für Wohlstand, das Bruttonationalprodukt, als unzureichend bezeichnete, da es die menschlichen Bedürfnisse nicht hinreichend abbildete. Der HDI setzt sich aus dem Lebensstandard, Bildungsindex, Lebenserwartungsindex und einem Einkommensindex zusammen (UNDP HDI 2016). Der Unterschied zwischen MPI und HDI besteht darin, dass der MPI seine Daten durch Haushaltsbefragungen erhält und daher absolute Zahlen angegeben werden können. Der MPI dient auch zur Lokalisation und Differenzierung von Armut, d.h. der Feststellung, wo welche Form der Armut auftritt (UNDP MPI 2016).

10 So entwickelte Thomas Pogge 1998 z.B. die globale Rohstoffdividende, um Armut abzuschaffen und zugleich einen gerechten Ressourceneinsatz einzuführen. Eine Rohstoffsteuer von $1 \%$ würde eine Dividende von 350 Milliarden US\$ abwerfen. Mit Hilfe dieses Geldes könnte Armut weltweit abgeschafft werden (gemäß UNO-Definition: weniger als 1 US\$ lokale Kaufkraft/Tag) (Pogge 1998). Die UN hat 2008 geschätzt, dass jährlich 30 Milliarden US-Dollar benötigt würden, um den Hunger auszurotten (Matthews 2008).

11 Jährlich sterben fast eine Million Menschen an Malaria, $75 \%$ von ihnen sind Kinder unter 5 Jahren. Schätzungen zeigen, dass durch Malaria jährlich etwa 12 Milliarden US-Dollar direkte Kosten entstehen. Die ökonomischen Verluste, die sich in geringeren Wachstumsraten des BIP niederschlagen, sind um ein Vielfaches höher (Center for Disease Control and Prevention 2016). Die ärmsten Länder können diese Summen nicht aufbringen, aber gerade die ärmsten Länder sind dem Malaria-Parasiten ausgeliefert. So sind 91\% der Todesfälle, die auf Malaria zurückzuführen sind, in Afrika zu lokalisieren. Die WHO hat 2007 berechnet, dass für eine effektive Malariabekämpfung südlich der Sahara zusätzlich zu den jährlich etwa 200 Millionen Euro externer Finanzmittel noch weitere 2 Milliarden Euro pro Jahr benötigt werden. Diese Gelder sollten von den Industrieländern weitgehend über den von UN-Generalsekretär Kofi Annan im Jahr 2000 initiierten „Global Fund to Fight HIV/AIDS, Tuberculosis and Malaria“ bereitgestellt werden (Müller 2007).

Bis heute hat die Bill-und-Melinda-Gates-Stiftung Fördergelder in Höhe von fast 2 Milliarden USDollar zur Bekämpfung von Malaria vergeben. Außerdem stellte sie dem Globalen Fonds zum Kampf gegen AIDS, Tuberkulose und Malaria 1,6 Milliarden US-Dollar zur Verfügung. Dies entspricht ungefähr 50\% der internationalen Finanzierung für die weltweite Malariakontrolle (Bill \& Melinda Gates Foundation 2016). Michael White et al. berechnen, dass „[t $\mathrm{t}$ he median financial cost of diagnosing a case of malaria was $\$ 4.32$ (range \$0.34-\$9.34). The median financial cost of treating an episode of uncomplicated malaria was \$5.84 (range \$2.36-\$23.65) and the median financial cost of treating an episode of severe malaria was \$30.26 (range \$15.64-\$137.87)“ (White et al. 2011). 
versorgen ${ }^{12}$ Wie viel würde es kosten, sicherzustellen, dass Menschen hinreichend mit Nahrungsmitteln versorgt sind und sie keine Mangelerscheinungen aufweisen? Das betrifft nicht nur den Kalorienmangel, sondern auch den Mangel an Eiweiß, Vitaminen, Ballaststoffen und wichtigen Spurenelementen.

Die Kriterien der Vereinten Nationen (Millennium Developmental Goals) ${ }^{13}$ klingen gut, ja, von humanistischem Geist getragen, wirken aber bei genauerer Betrachtung wie eine Kapitulationserklärung. Wie würde man darauf reagieren, wenn in einem Land wie zum Beispiel Deutschland ein Drittel der Menschen unterernährt wäre und davon wiederum ein größerer Teil diese Unterernährung mit dem Tod, mit Siechtum, mit Knochenschwund, mit chronischer Krankheit, mit Arbeitsunfähigkeit etc. bezahlen müsste? Man würde keine Zwanzigjahrespläne aufstellen, sondern sich sofort daranmachen, diesen Missstand zu beheben. Ein Konzept zur Reduzierung der Hungertoten um zehn Prozent im Jahr wäre in Deutschland völlig inakzeptabel. Genau dieser Logik folgen aber die MDGs. Sie setzen sich - bescheidene - Reduktionsziele, deren Bescheidenheit noch dadurch kaschiert wird, dass den Prozentzahlen nicht absolute Zahlen zugrunde gelegt werden, sodass bei Bevölkerungswachstum und gleichbleibendem Elend behauptet werden kann, die Reduktionsziele seien erreicht worden. ${ }^{14}$

12 Mehr als eine Milliarde Menschen haben keinen Zugang zu sauberem Wasser (WHO 2016). Um die MDGs zu erreichen bzw. einen universellen Zugang zu sauberem Trinkwasser zu ermöglichen, entstehen Kosten in Höhe von 30 Milliarden US\$ bzw. 6 Milliarden US\$ von 2010 bis 2015. 59 \% der Kosten entstehen in städtischen Gebieten und in Gebieten Subsahara-Afrikas (hier ist es v. a. der ländliche Raum), Südostasien, Westasien und Lateinamerika. Zieht man den notwendigen Ausbau von Sanitärbereichen in die Betrachtung mit ein, betragen die Kosten von 2010-2015 145 Milliarden US\$, wobei die höchsten Ausgaben in Subsahara-Afrika mit 53 Milliarden US\$ anfallen werden (Hutton 2012, S. 6).

13 Die Millennium Development Goals sind die acht Entwicklungsziele, welche im Jahr 2000 von den Vereinten Nationen eingeführt wurden und die es bis $2015 \mathrm{zu}$ erreichen galt. Alle $189 \mathrm{Na}$ tionen, die zu jenem Zeitpunkt Mitglieder der Vereinten Nationen waren, sowie 22 Institutionen verpflichteten sich, zur Erreichung dieser acht Ziele beizutragen:1) Abschaffung von Hunger und extremer Armut, 2.) Sicherstellung einer Grundschulbildung, 3.) Gleichstellung der Geschlechter und Stärkung der Rollen von Frauen, 4.) Senkung der Kindersterblichkeit, 5.) Verbesserung der Gesundheitsversorgung der Mütter, 6.) Bekämpfung von HIV, Malaria und anderen schweren Krankheiten, 7.) Ökologische Nachhaltigkeit und 8.) Entwicklung einer globalen Partnerschaft für Entwicklung (UN 2015). Im September 2015 wurden die MDGs auf dem Weltgipfel für nachhaltige Entwicklung 2015 von den 193 aktuellen Mitgliedstaaten der UN durch die 17 Sustainable Development Goals ersetzt. Nach diesen sollen nun bis 2030 weltweit Armut und Hunger verschwunden sein (UN 2016).

14 Zur methodischen Kritik an den MDGs vgl. Reddy/Pogge 2003; Koehler/Pogge 2015, S. 3-25; Batthyany 2015. 
Auch hier werden manche Leser einwenden, dies sei doch nicht vergleichbar, denn in Deutschland, diesem reichen, industrialisierten und mit einer hoch entwickelten sozialen Infrastruktur ausgestatteten Land, müsste das Auftreten chronischer Nahrungsmittelknappheit bei einem Teil der Bevölkerung selbstverständlich als Skandal gelten, ganz anders verhalte sich dies jedoch im globalen Rahmen. Aber überzeugt dieses Argument wirklich? Ich rede hier vom Elend der Milliarden, die von einer minimalen Kaufkraft existieren müssen, und das angesichts der gewaltigen wirtschaftlichen Dynamik in allen Regionen der Welt, auch in Afrika, auch in Ost- und Südasien, auch in Südamerika, mit einem kleinen Bruchteil des Weltsozialproduktes zu beheben wäre. Ich rede nicht von Hartz-IVSätzen, die zu globalisieren seien! Wenn eine deutsche Bundesregierung sich entschlösse, die Sozialhilfesätze zu halbieren, um Migranten abzuhalten, gäbe es zu Recht einen Aufschrei. Ja, es kann nicht sein, dass der ärmste Teil der Bevölkerung weiter in die Verarmung getrieben wird, das Bundesverfassungsgericht würde einschreiten, da solche Sozialhilfesätze nach ständiger Urteilspraxis des Bundesverfassungsgerichts die Menschenwürde verletzen. Dieses Niveau sozialer Absicherung zu globalisieren, mag unter den heutigen Bedingungen der Weltwirtschaft völlig unrealistisch sein. Eine solche sozialstaatliche Garantie erfordert hohe Steueraufkommen, eine funktionierende staatliche Sozialverwaltung und ein dichtes Kooperationsgefüge zwischen Staat, Wirtschaft und Gesellschaft, wie es heute nur in den ökonomisch entwickelten mittel- und nordeuropäischen Staaten realisiert ist. Ich rede hier von der Bekämpfung des bittersten Elends in der Welt, und nicht etwa von der Globalisierung des deutschen Sozialstaats.

Selbst die Konzentration des Vermögens bei den Reichsten der Welt interessiert mich in diesem Zusammenhang nicht. Gleichwohl ist beeindruckend zu erfahren, dass sich der Vermögenszuwachs in den USA, dem Land der weltweit meisten Milliardäre, ${ }^{15}$ seit Jahrzehnten auf die oberen ein bis drei Prozent der Bevölkerung konzentriert. ${ }^{16}$ Oder man betrachtet die globale Vermögensverteilung: In fast allen Staaten der Welt haben die Vermögens- und Einkommensungleichheiten ${ }^{17}$ in den vergangenen Jahrzehnten, man kann durchaus sagen, seit die neoliberale Agenda die nationalen und globalen Politiken und Praktiken zunehmend geprägt hat, massiv zugenommen. Nimmt man schließlich die Welt-

152015 wiesen 513 Individuen in den USA ein Privatvermögen von mehr als einer Milliarde US\$ auf (Credit Suisse 2015, S. 112).

16 In den USA konnten die reichsten 1\% ihren Einkommensanteil am Nationaleinkommen zwischen 1980 und 2014 verdoppeln (OXFAM 2016).

17 Schätzungen zufolge verfügt $1 \%$ der Weltbevölkerung über die Hälfte des Weltvermögens, ca. 110 Billionen US\$. Das ist $65 \mathrm{Mal}$ so viel, wie der unteren Hälfte der Weltbevölkerung zukommt (Dabla-Norris et al. 2015, S. 15). 
gesellschaft als Ganze in den Blick, ist dieser Effekt weit weniger stark ausgeprägt, was im Wesentlichen jedoch mit der Entwicklung im bevölkerungsreichsten Staat der Welt, in China, zusammenhängt. Bleibt China in den Statistiken außen vor, gilt auch für die Weltgesellschaft, dass die Ungleichheiten dramatisch zugenommen haben. ${ }^{18}$ Ungleichverteilungen sind aber per se ethisch irrelevant. Sie werden dann zum Gerechtigkeitsproblem, wenn sie zu Lasten der Schwächeren in der Gesellschaft gehen.

Im Rawls'schen Differenzprinzip gilt als Kriterium gerechtfertigter Ungleichheit, dass diese die Situation der am schlechtesten gestellten Personengruppe verbessert (genauer: so günstig wie nur möglich macht). Dies klingt paradox, ist es aber nicht. Manche Länder mit größeren Vermögens- und Einkommensunterschieden verzeichnen eine größere wirtschaftliche Dynamik, die auch den unteren Einkommensgruppen zugutekommen kann. Allerdings verdichten sich die Anzeichen, dass die dramatische Zunahme der Vermögens- und auch der Einkommensungleichheit ${ }^{19}$ weltweit dazu führt, dass die staatlichen Bildungssysteme erodieren, dass die Oberschichten und oberen Mittelschichten ihre Kinder nicht mehr in die staatlichen Schulen schicken, dass der Geldbeutel der Eltern immer einflussreicher auf die Bildungsentwicklung der Kinder wirkt, dass auf Eliteschulen die Kinder aus bestimmten Familien weitgehend unter sich sind,

18 Grundsätzlich wäre es möglich, dass die Ungleichheiten in allen Staaten deutlich zunehmen, ohne dass die Ungleichheiten der Weltgesellschaft zunehmen. Das könnte sogar mit einer Abnahme einhergehen, je nachdem, welche Länder welchen Zuwachs zu verzeichnen haben. Wenn die inneren Ungleichheiten zunehmen, aber die ärmeren und ärmsten Länder die stärksten Einkommens- und Vermögensgewinne zu verzeichnen haben, dann kann die Ungleichheit der Weltgesellschaft zurückgehen, obwohl die innerstaatlichen Ungleichheiten zunehmen.

19 In Deutschland ist die Vermögensungleichheit besonders hoch, vor allem dann, wenn man die Rentenansprüche nicht in die individuellen Vermögen einrechnet, wie das gegenwärtig praktiziert wird, während die Einkommensungleichheiten seit dem Wirksamwerden der Agenda-Reformen 2005 entgegen dem in den ökonomisch entwickelten Ländern zu beobachtenden internationalen Trend nicht mehr zugenommen haben (gemessen am Gini-Koeffizienten) und vergleichsweise moderat ausfallen. Nur die skandinavischen Länder und Kanada verzeichnen noch niedrigere Werte der Einkommensungleichheit (OECD 2016). Für Deutschland berechnet die OECD einen Wealth-Inequality-Index von 3,55, d.h., das ist das Verhältnis von Medianeinkommen zum Durchschnittseinkommen. Wenn dieser größer eins ist, heißt das, dass das Durchschnittseinkommen größer ist als das Medianeinkommen, was bedeutet, dass wenige Haushalte über ein hohes Vermögen und viele Haushalte über wenig Vermögen verfügen. Das DIW berechnet einen Gini-Koeffizienten von 0,78 für die Vermögensungleichheit in Deutschland (Grabka/Westermeier 2014, S. 156). Zugleich bewirken die progressiven Steuersätze eine recht deutliche Umverteilung hin zur Arbeitnehmerschaft, so dass Deutschland bei den Sekundäreinkommen zu den Ländern mit einer vergleichsweise moderaten Einkommensspreizung und hohen sozialen Mobilität gehört. 
dass eine neue Segregation, ja eine neue Klassengesellschaft, sich verfestigt. ${ }^{20}$ Es ist der Ausschluss der Vielen durch die zunehmenden ökonomischen Möglichkeiten der Wenigen, der uns Sorge bereiten muss. Zudem führen große Kapitalakkumulationen meist auch zu Möglichkeiten der politischen Einflussnahme, am Ende zu Systemen, wie man sie etwa in der Ukraine beobachten kann, wo wenige Oligarchen die politischen Geschicke des Landes steuern und dafür sorgen, daraus auch selbst ökonomischen Vorteil zu ziehen. Selbst die sympathische Variante der Verbesserung der Welt durch Milliardäre (Bill-und-Melinda-Gates-Stiftung etc.) ist ein Beitrag zur Refeudalisierung der Weltgesellschaft: Einige wenige Familien bestimmen über die Geschicke der Staaten und der Menschheit. Zudem trägt die globale Vermögenskonzentration zu vagabundierenden Kapitalien bei, die die Stabilität der Weltfinanzmärkte und der Weltwirtschaft als Ganze bedrohen. Der irritierende Konsens zwischen Links-Keynesianern und Finanzinvestoren, die für eine weitere Staatsverschuldung bei schon heute hohem globalen Niveau plädieren, verstärkt die Abhängigkeit der Weltgesellschaft von denjenigen, die über große Kapitalien verfügen. Es gibt ökonomische Interessen, die sowohl das japanische Modell der Staatsverschuldung bei der eigenen Bevölkerung als auch das deutsche Modell sinkender Staatsverschuldung und vermeintlicher Austeritätspolitik so unbeliebt machen: Es macht diese Staaten von den globalen Finanzmärkten und ihren Geldgebern unabhängiger.

Ungleichverteilung ist nicht per se ein Indiz für Ungerechtigkeit, sondern nur in der Folge einer Ungleichbehandlung von Individuen, oder wenn sie die ohnehin Benachteiligten weiter benachteiligt. Der gleiche individuelle Respekt, den Menschen verdienen, verlangt nach Gleichbehandlung als Bürger und als Menschen. Die Gleichbehandlung als Bürger wird im Idealfall durch die Bürgerrechte und ihre rechtsstaatliche Durchsetzung garantiert. Die Gleichbehandlung als Menschen verlangt nach einer gerechten Welt.

In der politischen Philosophie der Gegenwart werden Gerechtigkeitsfragen an institutionelle Strukturen gekoppelt. Anders formuliert: Viele zeitgenössische Philosophen meinen, dass man von Gerechtigkeit nur im Rahmen eines institutionell verfassten politischen Systems sprechen kann und nicht unabhängig davon. Die Erfordernisse der Gerechtigkeit richten sich demnach an diese Strukturen. Rawls spricht von der basic structure, die ganz unterschiedliche Institutionen beinhalten kann, wie etwa den ökonomischen Markt, den Sozialstaat oder Rechtsnormen. Um Konfusionen zu vermeiden, schlage ich vor, zwischen $\mathrm{Ge}$ rechtigkeit im weiteren Sinne und politischer Gerechtigkeit im engeren Sinne zu

20 Hierzu auch Federico Cingano: Trends in Income Inequality and Its Impact on Economic Growth (Cingano 2016). 
unterscheiden. Gerechtigkeitsfragen treten immer dann auf, wenn es um die relative Stellung von Individuen zueinander und den Einfluss geht, den unsere Praxis auf diese hat. Es ist ungerecht, den fünf Gästen auf einem Kindergeburtstag unterschiedlich große Kuchenstücke zu geben, wenn alle möglichst viel vom Kuchen abhaben wollen, außer es gibt dafür gute Gründe: Das eine oder andere Kind verträgt vielleicht nicht so viel Kuchen, man mag auch Differenzierungen nach dem Alter vornehmen oder nach der Körpergröße etc., fest steht aber, es ist ungerecht, wenn willkürlich eine Ungleichverteilung erfolgt. Hier ist die Machtstellung der Kuchen verteilenden Mutter des Geburtstagskindes irrelevant. Auch ein Kind, das selbst über keine Macht verfügt, würde die anderen Gäste der Party ungerecht behandeln, wenn es ohne Begründung den Kuchen ungleich aufteilt. Ungerechtigkeit verletzt, so könnte man sagen, den gleichen Respekt, der allen zukommt.

Politische Gerechtigkeit bezieht sich auf politische Praxis, und diese ist in der Regel an Institutionen gebunden. Der Versuch jedenfalls, Fragen der internationalen Gerechtigkeit dadurch abzublocken, dass man auf die fehlenden oder nur unzureichend entwickelten internationalen Institutionen verweist, kann nicht überzeugen. Eine Praxis ist ungerecht, wenn sie Menschen nicht den gleichen Respekt erweist, unabhängig davon, ob das Interaktionsgefüge institutionell verfasst ist oder nicht. Im Übrigen sollte man sich nicht täuschen, was die politische Gestaltung der Weltverhältnisse angeht. Die verschiedenen, schon abgeschlossenen oder in Verhandlung befindlichen weltweiten Handelsverträge bestimmen über die Verteilung von Reichtum und Armut mit. Manche Experten meinen, der gegenwärtig verhandelte Vertrag zwischen der EU und Westafrika würde, wenn er denn in Kraft tritt, die Fluchtursachen und damit den Strom der Menschen aus Regionen südlich der Sahara nach Europa verstärken und nicht abschwächen. Auch die Freihandelspolitik hat konkrete Verteilungswirkung. Umgekehrt können Zölle dazu beitragen, sich entwickelnde Länder des globalen Südens in Abhängigkeit und Armut zu halten. John Rawls war der Auffassung, dass sich seine Theorie der Gerechtigkeit, entgegen dem, was seine „Schüler“ Thomas Pogge und Charles Beitz, aber auch Brian Barry vorschlugen, nicht globalisieren lasse, weil sich zwar der Nationalstaat als ein System der Kooperation verstehen ließe, dessen Früchte dann fair zu verteilen wären, Gleiches aber für die Weltwirtschaft und die Weltgesellschaft nicht gelte, denn dort würden einzelne Nationalstaaten als Akteure agieren (Barry 1989; Beitz 1999; Pogge 1989). Dem ist allerdings entgegenzuhalten, dass schon in den 1990er Jahren, als John Rawls diese These näher in The Law of Peoples begründete, erst recht aber nach den weiteren Jahren der Globalisierung und internationalen Vernetzung, die Austauschbeziehungen so eng geworden sind, dass diese säuberliche Trennung von nationalstaatlichem Gerechtigkeitssinn und internationaler Gerechtigkeitsindif- 
ferenz nicht mehr überzeugen kann (Rawls 1999). Jedenfalls ist die Tatsache, dass Nationalstaaten ein komplexes politisches Institutionensystem entwickelt haben, zu dem es auf globaler Ebene keine Entsprechung gibt, kein hinreichender Grund, allenfalls einen ausgedünnten Gerechtigkeitsbegriff auf die globalen Verhältnisse anzuwenden.

\section{Bibliographie}

Barry, Brian (1989): Theories of Justice. Berkley: University of California Press.

Batthyany, Sacha (2015): „Das tägliche Sterben hält an“. Tages-Anzeiger vom 25. 09.2015.

https://www.tagesanzeiger.ch/ausland/das-taegliche-sterben-haelt-an/story/16782648, abgerufen am 04.12.2018.

Beck, Ulrich (2004): Der kosmopolitische Blick. Frankfurt am Main: Suhrkamp.

Beck, Ulrich/Beck-Gernsheim, Elisabeth (2011): Fernliebe. Lebensformen im globalen Zeitalter. Frankfurt am Main: Suhrkamp.

Beitz, Charles (1999): Political Theory and International Relations. Princeton: Princeton University Press.

Bill \& Melinda Gates Foundation (2016): „Malaria Strategic Overview“. http://www. gatesfoundation.org/en/What-We-Do/Global-Health/Malaria, abgerufen am 10.12.2018.

Bundesministerium für wirtschaftliche Zusammenarbeit und Entwicklung (2015): Agricultural Development Policy. A Contemporary Agenda. London: Overseas Development Institute.

Center for Disease Control and Prevention (2016): Impact of Malaria. https://www.cdc.gov/ malaria/malaria_worldwide/impact.html, abgerufen am 10.12.2018.

Cimadamore, Alberto/Koehler, Gabriele/Pogge, Thomas (Hrsg.) (2016): Poverty and the Millennium Development Goals. A Critical Look Forward. London: Zed Books.

Cingano, Federico (2014): Trends in Income Inequality and Its Impact on Economic Growth (= OECD Social, Employment and Migration Working Papers 163). Paris: OECD Publications Service.

Corak, Miles (2011): „Inequality and Occupy Wall Street 5: Decline of the American Dream“. https://milescorak.com/2011/11/17/inequality-and-occupy-wall-street-5-decline-of-theamerican-dream/, abgerufen am 12.12.2018.

Corak, Miles (2016): Inequality from Generation to Generation (= IZA Discussion Paper 9929). Bonn: IZA.

Credit Suisse (2015): „Global Wealth Databook“. http://publications.credit-suisse.com/tasks/ render/file/index.cfm?fileid=C26E3824-E868-56E0-CCA04D4BB9B9ADD5, abgerufen am 04.10.2016.

Dabla-Norris, Era/Kochhar, Kalpana/Suphaphiphat, Nujin/Ricka, Frantisek/Tsounta, Evridiki (2015): Causes and Consequences of Income Inequality: A Global Perspective (= IMF Staff Discussion Notes 15). Washington: International Monetary Fund.

Food and Agriculture Organization (2014): „World Food Situation“. http://www.fao.org/ worldfoodsituation/csdb/en/, abgerufen am 16.11.2018.

Food and Agriculture Organization (2015): „The State of Food Insecurity in the World 2015“. http://www.fao.org/hunger/en/, abgerufen am 16.11.2018.

Freeman, Samuel (Hrsg.) (2001): Collected Papers. Cambridge, MA: Harvard University Press. 
Grabka, Markus M./Westermeier, Christian (2014): „Anhaltend hohe Vermögensungleichheit in Deutschland“. In: Vermögensverteilung (= DIW Wochenbericht 9), S. 151-164.

Heidbrink, Ludger (2003): Kritik der Verantwortung. Zu den Grenzen verantwortlichen Handelns in komplexen Kontexten. Weilerswist-Metternich: Velbrück Wissenschaft.

Hutton, Guy (2012): Global Costs and Benefits of Drinking-water Supply and Sanitation Interventions to Reach the MDG Target and Universal Coverage. Genf: WHO.

Koehler, Gabriel/Pogge, Thomas (Hrsg.) (2015): Poverty and the Millennium Development Goals. A Critical Look Forward. London: Zed Books.

Maclntyre, Alasdair (1995): „Ist Patriotismus eine Tugend?“. In: Axel Honneth (Hrsg.): Kommunitarismus. Eine Debatte über die moralischen Grundlagen moderner Gesellschaft. Frankfurt am Main: Suhrkamp, S. 84-102.

Matthews, Christopher (2008): „The World Only Needs 30 Billion Dollars a Year to Eradicate the Scourge of Hunger“. http://www.fao.org/NEWSROOM/en/news/2008/1000853/index. html, abgerufen am 09.11.2016.

Miller, David (1995): On Nationality. New York: Oxford University Press.

Müller, Olaf (2007): „Im Kampf gegen die Malaria“. Forschung - Das Magazin der Deutschen Forschungsgemeinschaft 2007/1, S. 4-7.

Nagel, Thomas (2005): „The Problem of Global Justice“. In: Philosophy and Public Affairs 33, S. $113-147$.

Nida-Rümelin, Julian (2006): Demokratie und Wahrheit. München: Beck.

Nida-Rümelin, Julian (2011): Die Optimierungsfalle: Philosophie einer humanen Ökonomie. München: Irisiana.

Nida-Rümelin, Julian (2017): Über Grenzen denken: Eine Ethik der Migration. Hamburg: Körber. Nida-Rümelin, Julian (2018): Structural Rationality and other Essays on Practical Reason. Berlin, New York: Springer.

OECD (2016): „Income Distribution and Poverty“. http://stats.oecd.org/Index.aspx? DataSetCode=IDD, abgerufen am 04.10.2016.

OXFAM (2016): „Working for the Few. Political Capture and Economic Inequality“. https://www. oxfam.de/system/files/bp-working-for-few-political-capture-economic-inequality-200114en-oxfam.pdf, abgerufen am 04.10.2016.

Pogge, Thomas (1989): Realizing Rawls. Ithaca: Cornell University Press.

Pogge, Thomas (1998): „A Global Resources Dividend“, In: David Crocker/Toby Linden (Hrsg.): Ethics of Consumption. The Good Life, Justice, and Global Stewardship. New York: Rowman \& Littlefield, S. 501-536.

Pogge, Thomas (2003): „The First UN Millennium Development Goal: A Cause for Celebration? Oslo Lecture in Moral Philosophy, 11.09.2003“. Oslo: University of Oslo.

Rawls, John (1993): Political Liberalism. New York: Columbia University Press.

Rawls, John (1999): The Law of Peoples. Cambridge, MA: Harvard University Press.

Reddy, Sanjay/Pogge, Thomas (2003): „How Not to Count the Poor“. New York: Barnard College.

Sandel, Michael (1982): Liberalism and the Limits of Justice. Cambridge: Cambridge University Press.

UN (2015): „Millennium Development Goals and Beyond“. http://www.un.org/millenniumgoals, abgerufen am 05.12.2018.

UN (2016): „Sustainable Development Goals“. http://www.un.org/sustainabledevelopment/ sustainable-development-goals/, abgerufen am 15.12.2018. 
UNDP HDI (2016): „Human Development Reports“. Human Development Index (HDI). http://hdr. undp.org/en/content/human-development-index-hdi, abgerufen am 12.12.2018.

UNDP MPI (2016): „Human Development Reports“. The 2016 Global Multidimensional Poverty Index (MPI). http://hdr.undp.org/en/content/multidimensional-poverty-index-mpi, abgerufen am 12.12.2018.

UNICEF/WB/WHO/UN (2015): Levels and Trends in Child Mortality. Report 2015.

Walzer, Michael (1983): Spheres of Justice. A Defense of Pluralism and Equality. New York: Basic Books.

Weltagrarbericht (2013): Wege aus der Hungerkrise: Die Erkenntnisse und Folgen des Weltagrarberichts: Vorschläge für eine Landwirtschaft für morgen. Berlin: Zukunftsstiftung Landwirtschaft.

White, Michael/Conteh, Lesong/Cibulskis, Richard/Ghani, Azra G. (2011): „Costs and Cost-effectiveness of Malaria Control Interventions - A Systematic Review“. In: Malaria Journal 10. Nr. 337, https://www.ncbi.nlm.nih.gov/pmc/articles/PMC3229472/, abgerufen am 10.12.2018.

WHO (2016): „Drinking-water Fact Sheet“. http://www.who.int/mediacentre/factsheets/fs391/ en/, abgerufen am 10.12.2018.

World Food Programme (2013): „Hunger ist kein Schicksal“. In: Eine-Welt-Presse. Nord-Süd-Zeitung der Deutschen Gesellschaft für die Vereinten Nationen (DGVN) e.V., S. $1-8$.

World Bank (2017): „WB: World Development Indicators. Nutrition and Growth“. http://wdi. worldbank.org/table/2.18, abgerufen am 10.12.2018.

World Health Organization (2016): World Health Statistics 2016: Monitoring Health for the SDGs. 